\title{
IMPLICANCIAS DEL MÉTODO DE COSTEO ABC
}

\author{
IMPLICATIONS OF ABC COSTING METHOD \\ BERNARDO SÁNCHEZ BARRAZA* \\ Docente Asociado de la Facultad de Ciencias Contables \\ Universidad Nacional Mayor de San Marcos-UNMSM / Lima-Perú \\ [Recepción: Mayo de 2013/ Conformidad: Junio de 2013]
}

\section{RESUMEN}

Toda empresa para competir con éxito en el campo empresarial, necesita precisar con exactitud cuáles son sus costos de producción, porque ellos determinarán el nivel de ingreso y medición de ganancias en las empresas. Los métodos de costeo tradicionales se encuentran hoy cuestionados, por que se afirma que no asignan con precisión los costos a los productos. Ante esta problemática, surge el método de costeo $\mathrm{ABC}$ (Activity Based Costing) como un método de asignación de costos más realista y exacto, por ello en nuestra investigación, realizaremos una revisión teórica de estos métodos de costeo. La motivación de esta investigación es porque pensamos que el sector industrial peruano fabrica productos de calidad internacional, pero para lograr un adecuado desarrollo de este sector, las empresas industriales necesitan saber sus costos exactos para ver si son competitivos en comparación con potencias industriales a nivel mundial. Finalmente, de esta investigación estamos seguros que se generarán nuevos conocimientos de costos que serán de utilidad al lector que pueda tener similares problemas en cualquier rubro empresarial.

Palabras clave: Costos de producción, método de costeo $\mathrm{ABC}$, método de costeo tradicional, empresa industrial

\begin{abstract}
Every company to compete successfully in their business, need to know exactly what are their costs of production, because they determine the level of income and measurement profits of the companies. The traditional costing methods are challenged today by stating that not accurately assign costs to products. Faced with this problem, there is the $\mathrm{ABC}$ costing method (Activity Based Costing) as a cost allocation method is more realistic and accurate, so in our research, we will carry out a theoretical review of these costing methods. The motivation of this research is that we think the Peruvian industrial sector manufactures products of international quality, but to achieve an appropriate development of this sector, industrial enterprises need to know their exact costs to see if they are competitive in comparison with industrial powers at the global level. Finally, we are confident that this research will generate new knowledge of costs that will be useful to the reader who may have similar problems in any business item.
\end{abstract}

Keywords: production costs, $\mathrm{ABC}$ costing method, traditional costing method, industrial company

\footnotetext{
* Contador Público Colegiado Certificado, Maestrante en Contabilidad de Gestión. E-mail: jsbjsb20042004@yahoo.com/sanchez.b@pucp.pe
} 


\section{INTRODUCCIÓN}

Antiguamente se pensaba que los productos o servicios eran los causantes de los costos. Respecto a los costos de materia prima directa y mano de obra directa para fabricar un producto, se puede afirmar que no existe problema para asignar los costos, debido a que ellos son directos a los productos. Sin embargo para fabricar un producto también se necesita de los costos indirectos, estos al ser indirectos son de difícil asignación a los productos. Tradicionalmente estos costos indirectos se asignaban a los productos de acuerdo a una tasa de asignación en base a las horas hombre consumidas en la elaboración del producto, pero ello no reflejaba un cálculo exacto en los costos porque algún producto que no era intensivo en horas hombre recibía pocos costos indirectos, sin analizar si realmente ese producto consumió mayor o menor proporción de los costos indirectos. Ante esta problemática, surge el Método de costeo $\mathrm{ABC}$ (Activity Based Costing), el cual plantea que no son los productos los que generan costos, si no las actividades necesarias para la fabricación de un producto, son las que realmente consumen recursos y por lo tanto generan costos. De esto se desprenden que a los productos se les asignará los costos de las actividades, solo si ellos pasaron por dichas actividades para ser fabricados, por lo tanto un producto que no atraviese alguna actividad no deberá recibir ningún costo de esa actividad. Elegimos investigar el tema de costeo $\mathrm{ABC}$ porque es una herramienta que ayuda a realizar una asignación de costos a los productos más exacta y fiable de ahí es que también radica su importancia para una adecuada toma de decisiones gerenciales en base a costos más fidedignos.

\section{BASES TEÓRICAS DEL MÉTODO DE COS- TEO ABC}

\section{*Antecedentes del Costeo ABC}

El Costeo ABC significa Activity Based Costing por sus siglas en inglés, lo cual puede traducirse en Costeo Basado en las Actividades. Este método fue desarrollado por los profesores Robert S. Kaplan y Robin Cooper de la Universidad de Harvard en los Estados Unidos de Norteamérica.
Según Bellido, en el año 1972 fue creada en U.S.A una organización sin fines de lucro que agrupa industriales, consultores y otros, denominada Manufactura Asistida por Computadora Internacional (Computer Aided Manufacturing - International CAM-I), la cual aportó a través del programa CMS los conceptos fundadores del $\mathrm{ABC}$, estos conceptos fueron publicados en asociación con Harvard Bussines School una obra titulada Gerencia de Costos para la Manufactura de hoy. El diseño conceptual de CAM-I.

A partir de los años 80, comienza a proliferar la literatura sobre este tema, tal es el caso de la obra The Goal, de los autores E. Goldratt y J. Cox (1984) donde se critican las fallas del costeo tradicional, dando paso en los años siguientes, a una nueva corriente de pensamiento en cuanto a los métodos para calcular costos y determinar precios. Dado este fenómeno, el profesor Robert S. Kaplan de la Harvard Business School, propone la metodología $\mathrm{ABC}$ que se fundamenta en una hipótesis básica: las distintas actividades que se desarrollan en las empresas son las que consumen los recursos y las que originan los costos, no los productos; estos solo demandan las actividades necesarias para su obtención. Esta propuesta se ve ampliada en el interesante libro escrito en el año 1987 por los profesores Robert S. Kaplan y H. Thomas Johnson, titulado Relevancia perdida: Auge y Caida de la Contabilidad Gerencial (Relevance Lost: The Rise and Fall of Management Accounting. Boston, Harvard Bussines School Press).

Por otro lado, según Douglas Hicks, a finales de los ochenta del siglo XX, organizaciones como CAM-I y la National Association of Accountants introdujeron el sistema de cálculo de costos basado en actividades. En 1987 el profesor adjunto de Harvard, Robin Cooper, escribió el artículo ¿Necesita su compañía un nuevo Sistema de Costos? Luego en febrero de 1988, Robert S. Kaplan escribió el artículo "Un sistema de costos no es suficiente, donde se expresa que los sistemas de costos tradicionales son inadecuados. En abril de 1988 Cooper y Kaplan escriben conjuntamente el artículo "La Contabilidad de Costos distorsiona los costos de los productos".

66/ QVIPURAMAYOC | Vol. 21(39) 2013 
Finalmente estos dos profesores Cooper y Kaplan publican otro artículo llamado "Costeo Basado en Actividades" publicado en la Harvard Bussines Review de fecha Mayo-Junio 1991, donde se explica claramente los enfoques del Sistemas de Costos Tradicionales y el $\mathrm{ABC}$.

\section{${ }^{*}$ Gestión Basada en Actividades (Activity Based Management - ABM)}

Se define como la filosofía de las actividades, la cual reconoce que toda empresa tiene un número de actuaciones superfluas y por consiguiente derroche de dinero. Esto determina que las empresas tienen un potencial de mejora continua, lo cual se conseguirá si se pueden reducir las tareas o actividades superfluas que consumen recursos. Debemos destacar que el término superfluo se refiere a que no brinda valor agregado para el objeto de costo que la empresa ofrece a los clientes. Algo importante en el ABM es la inclinación a la gestión de las actividades, en lugar de la gestión de los costos, porque si se llega a generar una información adecuada de las actividades superfluas, se podrá conseguir una importante reducción de actividades y de sus costos.

Debemos hacer una distinción entre el ABM y el $A B C$, el $A B M$ tiene una orientación a lograr una excelencia empresarial en la gestión de la empresa, en cambio el $\mathrm{ABC}$ tiene una dimensión contable y numérica, la cual apoya a los objetivos generales que persigue el ABM. En general, ambos términos pretenden obtener información acerca de las causas que originan la realización de un trabajo o actividad, es decir el "inductor de actividad", así como analizar la forma en la que se llevan a cabo o las "medidas de ejecutar o realizar".

\section{${ }^{*}$ Costeo Basado en Actividades (Activity Based Costing - ABC)}

El Método de Costeo ABC analiza las actividades de los departamentos indirectos dentro de una institución para calcular el costo de los productos terminados. Además plantea un análisis de las actividades.

Los principios sobre los cuales está basado el $\mathrm{ABC}$ son los siguientes:
- No son los productos los que consumen los recursos de la empresa si no las actividades.

- Los productos consumen actividades.

- La gran mayoría de los costos (gastos) indirectos se consideran generalmente como fijos (dentro del rango relevante, además pueden ser costos escalonados).

- No varían según los volúmenes de producción sino según los niveles de las actividades.

Por lo tanto este método tiene dos ejes vertebrales muy importantes, los cuales se definen de la siguiente manera:

1) No son los productos sino las actividades las que causan los costos.

2) Son los productos los que consumen las actividades

Estos enfoques plantean que los costos se relacionan con los productos por medio de las actividades, las cuales son causa de los primeros y son consumidas por los segundos.

$\mathrm{Al}$ referirse al Método de Costeo ABC, el autor Romero sostiene que en primer lugar, no son los productos sino las actividades las que causan los costos y, en segundo lugar, son los productos los que consumen las actividades. Así es precisamente como se ligan los costos con los productos: por medio de las actividades, las cuales son causa de los primeros y son consumidas por los segundos.

En cuanto al mismo término, Horngren manifiesta que los sistemas de costeo basados en actividades (CBA) perfeccionan los sistemas de costeo al concentrarse en actividades individuales como objetos del costo fundamentales. Los sistemas de CBA calculan los costos de actividades individuales y asignan los costos a objetos del costo, tales como productos y servicios, sobre la base de las actividades realizadas para producir cada producto o servicio.

Debemos enfatizar que el Método de Costeo $\mathrm{ABC}$ se encuentra enmarcado en la "filosofía de las actividades", la que plantea que las empresas tienen actuaciones (actividades) superfluas y por consiguiente derroche de recursos. Las actividades superfluas no generan valor agregado a los objetos de costeo (productos/servicios) desde el punto de vis- 
ta de calidad del producto, a su vez no será atractivo para el cliente. Por lo tanto, es necesaria una orientación hacia la gestión de las actividades que solo añaden valor, esta reducción de actividades desembocará en una reducción de costos en las empresas. A toda esta filosofía se le denomina, Gestión Basada en Actividades (Activity Based Management) por ello antes de aplicar numéricamente el Método de costeo $\mathrm{ABC}$ debemos idear y planificar los conceptos de costos según la Gestión basada en actividades y luego plasmarlos con el citado método $\mathrm{ABC}$.

\section{DIMENSIONES PARA MÉTODO DE COSTEO ABC}

\section{Actividades}

De forma genérica el término "actividad" viene del latín activitas que significa 'actuar'. Por otro lado las actividades son tareas que realizan las personas para la obtención de un bien o servicio.

Las actividades en el campo industrial se relacionan en conjuntos o grupos que forman el total de procesos productivos, los cuales son ordenados de forma lógica, secuencial y simultánea para obtención de productos con valor agregado que le adicionan en cada proceso. Finalmente, la realización de actividades consume recursos de la empresa que se acumulan por medio de los costos.

A continuación se expondrá otras definiciones del término actividad de diversos autores de costos:

Según Horngren define una actividad se define como un acontecimiento, tarea o unidad de trabajo que tiene un motivo específico; por ejemplo el diseño de productos, la preparación de las máquinas, la operación de las máquinas y la distribución de los productos. Respecto al mismo término, Álvarez enfoca que una actividad es un conjunto de actuaciones o de tareas que tienen como objetivo la atribución, al menos a corto plazo, de un valor añadido a un objeto (producto o proceso) o al menos permitir añadir este valor, bajo la perspectiva del cliente o usuario del mismo.

En conclusión las definiciones de actividad de los dos autores descritos tienen similar concepto en su esencia.

\section{Cost Driver (Medida de actividad)}

Los cost drivers son medidas de competencia que se utilizan como un nexo entre las actividades de una empresa y los costos (gastos) indirectos de fabricación que ellas puedan generar, por otro lado estas medidas de actividad deben poder relacionarse con los productos fabricados, para tal efecto las medidas de actividad deben definirse en unidades de actividad identificables con una relación causaefecto. Existen una serie de definiciones de expertos en costos sobre el término cost driver, sin embargo las más claras y utilitarias son las siguientes, a la luz del estilo propio de los autores:

El autor Romero conceptualiza que las "medidas de actividad" son medidas competitivas que sirven como conexión entre las actividades y sus gastos indirectos de fabricación respectivos y que se pueden relacionar también con el producto terminado. Cada medida de actividad debe estar definida en unidades de actividad perfectamente identificables. Dichas medidas de actividad son conocidas como Cost Drivers, término cuya traducción en castellano aproximada sería la de origen del costo (o manejador del costo), porque son precisamente los cost drivers los que causan que los gastos indirectos de fabricación varíen. Por otro lado, Álvarez hace una definición interesante y concluyente, en cuanto a que expresa que la "Unidad de Actividad" constituye la variable que permite cuantificar las realizaciones de las distintas actividades. Se trata pues, de establecer una relación de causalidad, para cada actividad, entre el consumo de los recursos y la producción obtenida. Este concepto de producción no debe estar relacionado necesariamente con un producto, sino que estará vinculado a un concepto genérico de output.

En nuestra opinión podemos afirmar que los cost driver o medidas de actividad deben hacer uso de las unidades de actividad para de esta manera obtener un costo más preciso de los bienes o servicios (output) que se pretendan obtener en una empresa, mediante la relación causa y efecto. 


\section{METODOLOGÍA DEL COSTEO ABC}

Existen diferentes enfoques de abordar los pasos o procedimientos para implementar o poner en marcha el Costeo $\mathrm{ABC}$, de forma práctica o sencilla podemos resumir lo siguiente:

Paso 1-Recursos: Determinar e identificar cuales son los recursos que la empresa consume para la realización de su misión y actividades que proveen los departamentos. Estos recursos deben estar expresados en unidades monetarias.

Paso 2-Actividades: Identificar las actividades que efectúa la empresa para el logro de sus objetivos, para tal efecto se debe realizar un "mapa de actividades", pero solo de las que brinda un valor agregado al producto o servicio. Esto implica que se deben eliminar o suprimir las actividades que no dan valor agregado. Finalmente, se debe asignar a cada actividad los costos que han consumido, de esta forma se crean grupos de costos homogéneos en los que el comportamiento de todos los costos de cada grupo es explicado por una misma actividad.

Paso 3-Cost driver de recursos: Establecer la forma en que las actividades consumen recursos en la empresa, es decir buscar las medidas de competencia que se utilizan como un nexo causal entre las actividades y los recursos.

Paso 4-Medidas de Actividad: Se deben encontrar las "medidas de actividad" que mejor expliquen el origen y variación de los costos indirectos de fabricación. Las medidas de actividad son conocidas como cost driver (origen del costo). Mientras más unidades de actividad del cost driver se consuma, mayores serán los costos indirectos consumidos con esa actividad.

Paso 5-Costo unitario por actividad: Se debe calcular el costo unitario de proveer cada actividad al proceso productivo, para tal efecto, se divide el costo indirecto total de una actividad entre el número de "unidades de actividad" consumidas de la "medida de actividad" o "cost driver identificado".

En resumen se emplea la siguiente fórmula:

Costo unitario por Actividad $=$ Costos indirectos totales de la actividad / Número total de "unidades de actividad" del "cost driver" específico.

Paso 6-Productos/Servicios: Identificación de los productos o servicios que se benefician con las actividades o que gracias a ellas se fabrican los productos o se prestan los servicios.

Paso 7-Cost driver de actividades: Determinar cómo los productos o servicios consumen los recursos o costos de las actividades, es decir se debe identificar el número de "unidades de actividad" consumidas por cada producto en su fabricación.

Paso 8-Costeo: Costear los recursos de las actividades a los productos o servicios que pasen por dichas actividades. Para tal efecto, se deben asignar los costos indirectos a los productos, esto se logra multiplicando el costo unitario de proveer cada actividad por el número de unidades de actividad requeridas para cada producto en su elaboración.

En este caso se emplea la siguiente fórmula:

Costo indirecto de actividad a asignar a cada producto $=$ Costos unitario por unidad de actividad $x$ Número de unidades de actividad consumidas por un producto

El siguiente esquema resume como se asignan los recursos de una empresa a las actividades y que finalmente son desembocados en los productos que consumen las actividades.

Finalmente, debemos ser conscientes que para elegir una adecuada clasificación de actividades en una empresa que aplicará el método de costeo $\mathrm{ABC}$, debe tenerse en cuenta el rubro empresarial al que

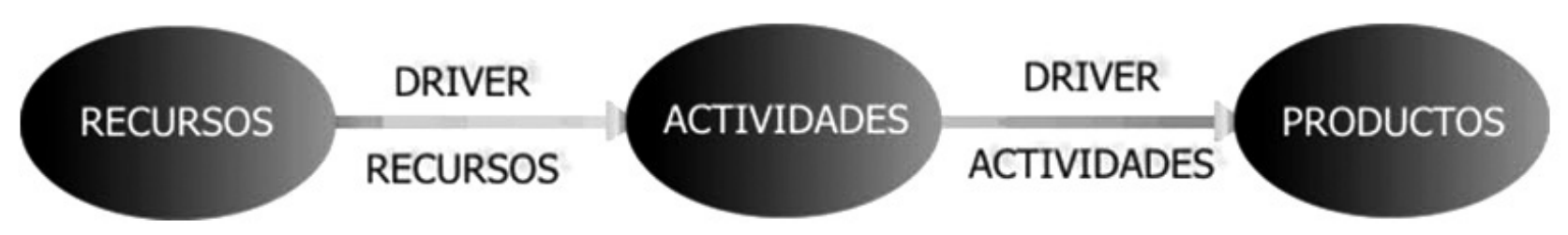

Vol. 21(39) 2013 | QVIPURAMAYOC /69 
pertenece la empresa y la simplificación de las actividades, para así lograr un ahorro de tiempo y dinero en la implementación del costeo.

\section{BASES TEÓRICAS DEL MÉTODO DE COS- TEO TRADICIONAL}

Los métodos de costeo que se han utilizado tradicionalmente correspondían a una necesidad de competir en mercados limitados y con productos muy concretos o estandarizados, muchos de ellos expresaban la producción de un solo producto.

Por lo tanto los procesos productivos estandarizados y especializados permitían la elaboración de productos altamente homogéneos y en grandes volúmenes productivos. Pero la variedad de productos que se podían obtener respecto a una estructura productiva común planteó la distinción de costos directos (las cuales permiten la obtención del producto) y costos indirectos (generados por el mantenimiento de una capacidad productiva).

Según Álvarez, el sistema tradicional de cálculo de costo se había organizado así en torno a los procesos de producción, dando lugar al denominado método de las secciones homogénea, en virtud del cual los costos directos (los materiales y en gran medida la mano de obra) se asignan directamente a los productos, mientras que los costos indirectos deben circular y acumularse en las distintas secciones o centros de costes antes de ser imputados a los productos; llevado a cabo a través de una unidad de obra, que representa la prestación realizada por la sección y consumida por el producto. La unidad de obra de cada sección constituye así una simplificación y una aproximación en el proceso real de causalidad del consumo de los recursos.

Este sistema de costo sienta sus bases en la idea de que el producto fabricado es el que causa el consumo de diferentes recursos productivos, por lo tanto se puede establecer la relación causal:

Volumen de producción (causa) ----->Costos

Finalmente, el costeo tradicional suele ser enfocado con una sola causalidad de los costos indirectos de fabricación, es decir emplea para el costeo una "tasa única", la cual usualmente ha estado expre- sada en términos de horas hombre o unidades físicas producidas del bien.

\section{Dimensiones para el Método de costeo tradicional}

\section{Volumen productivo}

Es el nivel de productos o artículos que fabrica una empresa industrial o nivel de prestación de servicios de una empresa. El volumen productivo puede expresarse en unidades "físicas" de producto (botellas, cajas, piezas, etc.) o en unidades de medida como galones, kilogramos, toneladas, litros, etc. En los servicios se pueden emplear horas, capacidad de aulas, número de atención de clientes, etc. Por otro lado el volumen productivo se encuentra inmerso dentro del concepto de "capacidad", debido a que se considera que el volumen productivo representa el consumo de la capacidad de planta que tiene una empresa.

En cuanto a este tema, Álvarez esclarece este concepto afirmando que la capacidad hay que entenderla como la cantidad fija con que contamos de planta industrial, equipo productivo y elemento humano, que son necesarios para desarrollar en las óptimas condiciones el proceso de producción. La capacidad es la posibilidad que tiene la empresa para obtener una producción (servicios o productos) y el nivel de actividad es la utilización que hacemos de la capacidad.

En la cita precedente debemos indicar que el nivel de actividad es sinónimo de volumen de producción real que alcanza una empresa en un periodo establecido.

\section{Indicadores del volumen productivo}

Número de unidades de producto: se refiere a la cantidad de productos que se están fabricando. Ejemplo: 500 litros de jugo, 300 pantalones.

Número de horas de producción: alude al tiempo empleado en la producción. Ejemplo: la fabricación de 1000 camisetas necesitó 2000 horas de producción.

\section{Procesos productivos}

Se define como aquella serie de pasos y acciones que realizan las personas con ayuda de herramientas y maquinarias para lograr la transformación de 
materias primas en productos terminados o se brindan o realizan servicios de cualquier índole.

El término proceso se emplea en conceptos relacionados como "costeo por procesos", para completar nuestra definición vemos que Polimeni argumenta que el costeo por procesos es un sistema de acumulación de costos de producción por departamento o centro de costos. Un departamento es una división funcional principal en una fábrica donde se realizan procesos de manufactura relacionados.

En conclusión los procesos productivos son etapas productivas por las cuales atraviesa un producto hasta lograrse como un producto totalmente terminado y listo para la venta.

\section{Dimensión de procesos productivos}

Las dimensiones para los procesos productivos serían el definir las labores que realiza cada proceso productivo.

\section{Indicador de procesos productivos}

Números de procesos productivos: puede ser que un producto necesite tres procesos productivos como proceso de corte, proceso de cocción y proceso de empaque. Otro producto puede necesitar unos 50 procesos como el caso de la fabricación de llantas para automóviles.

\section{Costos}

Son los valores monetarios sacrificados por los consumos de recursos (materiales, personal, energía, etc.) para la obtención de un objeto de costo (producto o servicio), el cual pensamos nos brindará un beneficio presente o futuro. En cuanto a este término Polimeni define de forma precisa el concepto puro de costo, como el valor sacrificado para adquirir bienes o servicios, que se mide en dólares mediante la reducción de activos o al incurrir en pasivos en el momento en que se obtienen los beneficios. Por consiguiente el concepto de "costo" involucra un sacrifico de dinero que luego se verá recompensado por la generación de beneficios.

\section{Ingresos}

Es el dinero que se genera de la venta de un producto o servicio. Es importante indicar que el ingreso debe ser suficiente para cubrir el costo de un producto o servicio y a su vez quede un margen de ganancia por el negocio realizado. Citando al autor precedente se define el término ingreso como el precio de productos vendidos o de los servicios prestados.

En Perú el término "precio" contiene el Impuesto General a las Ventas (IGV), el cual para determinación del ingreso que se muestra en el Estado de Resultados Integrales (Estado de Ganancias y Pérdidas) deber ser extraído; debido a que el IGV, no es costo ni gasto para la empresa, ello se debe a su naturaleza de trasladable y por pertenecer al fisco. Cabe destacar que a nivel internacional los conceptos similares al IGV son el IVA (impuesto sobre el valor agregado) y el Tax (aplicado en U.S.A.).

\section{Dimensión de Costos e Ingresos- Recursos con- sumidos y Recursos recibidos}

La dimensión para los costos serían los montos de dinero que representan los consumos de recursos efectuados por las empresas. En cuanto a la dimensión para los ingresos serían los montos de dinero recibido por ventas de productos o servicios.

\section{Indicador de Costos e Ingresos}

Se expresa en la unidad monetaria de curso legal en el Perú, (S/.) Nuevos soles, vigente desde 1991.

\section{Costos de las actividades}

Los costos de las actividades están determinados por todos los costos de materias primas, mano de obra y costos indirectos de fabricación que se necesitan para la realización de una o más actividades.

\section{Dimensión de Costo de las actividades- Recur- sos consumidos}

Son los montos de dinero que representan los consumos de materias primas, mano de obra y costos indirectos de fabricación. Ejemplo: consumo de azúcar, salario de un trabajador y agua para la fabricación de un jugo envasado.

\section{Indicador del Costos de las actividades}

Se expresa en la unidad monetaria de curso legal en el Perú, (S/.) Nuevos soles, vigente desde 1991.

\section{Productos Rentables}

Son los productos que generan un ingreso por venta mayor que su costo de producirlo. El ingreso de un producto contiene una ganancia por el esfuer- 
zo o valor agregado que realiza la empresa al fabricar un artículo. La "utilidad" que genera un producto rentable se denomina "ganancia".

\section{Productos Deficitarios}

Son los productos que generan un ingreso por venta menor que su costo de producirlo. La "utilidad" que genera un producto deficitario se denomina "pérdida". Debemos tener cuidado que el término "utilidad" no necesariamente involucra un concepto de ganancia.

\section{ALGUNAS HISTORIAS DE ÉXITO, BENEFI- CIOS Y LIMITACIONES DEL COSTEO ABC}

Según Apaza a nivel internacional podemos citar como historias de éxito de implementación del Costeo $\mathrm{ABC}$ en Chrysler Corporation donde los ahorros se estiman en centenares de millones de dólares. En cuanto a la empresa Sfety-Kleen ha obtenido ahorros de más de $\$ 12,7$ millones, aquí el $\mathrm{ABC}$ fue utilizado para reducir líneas de productos, racionalizar operaciones y ampliar nuevos mercados.

\section{Beneficios:}

- Facilita el costeo justo y realista por línea de producto, en especial a los no relacionados con volúmenes.

- El costeo $\mathrm{ABC}$ reconoce la relación causal entre generadores de costos y actividades de producción.

- Analiza diversos objetos del costo, como servicios y actividades, además del producto físico.

- Brinda datos financieros y no financieros para la gestión y control de los costos.

- Ayuda a la identificación, al rastreo y prorrateo de los costos.

\section{Limitaciones:}

- No toma en cuenta las consecuencias del comportamiento humano y organizacional (reducción de actividades puede significar despido de recursos humanos).

- La información obtenida es histórica y devengada.

- La búsqueda, identificación y selección de los cost-drivers y costos comunes no son fácil de determinar y relacionar de forma infalible.
- El ABC se ve influenciado por juicios cualitativos y decisiones, por ello es un poco incierto su aporte en áreas de medidas y control.

- Cuando ya es implementado se tiende a dejar de motivar a la mejora continua y al cambio, además no contempla la satisfacción del cliente final.

\section{Conclusiones y recomendaciones}

- La implicancia o consecuencia del Costeo ABC en comparación con el Costeo Tradicional permite una identificación de productos rentables y deficitarios o menos rentables, lo cual se podrá comprobar implementado la metodología expuesta. Esta metodología se pondrá en práctica en un posterior artículo de nuestra revista.

- El costeo ABC al obtener cálculos más exactos del costo de productos, permite tomar mejores decisiones respecto a la evaluación de la rentabilidad, inversión, supresión de productos entre otros aspectos de gestión contenidos en el Estado de Situación Financiera (Balance General), concretamente en el elemento 2 de dicho estado.

- El uso del costeo ABC no solo debe ser enfocado desde el punto de vista numérico y de método de costeo, sino debe ser trabajado a la luz de la filosofía del ABM para lograr un verdadero ahorro de costos al eliminar actividades superfluas que no dan valor agregado a los productos o servicios.

- Debemos ser conscientes que el método de Costeo $\mathrm{ABC}$ no puede ser utilizado a nivel tributario en el Perú, debido a que la Ley del Impuesto a la Renta (Ley $N^{\circ} 29645$ ) plantea la determinación del cálculo de impuestos vía el Costeo Tradicional, es decir el Costeo por Absorción (Costeo Total o Costeo Tradicional).

- Las grandes empresas multinacionales se han desarrollado y obtenido buenas rentabilidades a la luz de la contabilidad de gestión, la cual propone el uso del Costeo $\mathrm{ABC}$ y del $\mathrm{ABM}$ para toma de decisiones gerenciales.

- Las Normas Internacionales de Información Financiera (NIC 2) no mencionan el método de costeo $\mathrm{ABC}$ dentro de su real dimensión e importancia; porque en ellas solo mencionan la medición de 
los inventarios al costo de adquisición y/o transformación o al valor neto realizable, según cuál sea menor. Como técnicas de medición de inventarios reconocen a los costos conjuntos, al método del costeo estándar y al método de los minoristas.

- Finalmente, los profesionales contables, de costos y usuarios de diferentes carreras profesionales que usan y se sirven de los informes de costos, deben reconocer la importancia del Costeo $\mathrm{ABC}$ y del $\mathrm{ABM}$ como una herramienta que permite el cálculo más realista de los costos y difundir su uso, al igual que los colegios profesionales y universidades públicas y privadas de nuestro país, deben adquirir ese compromiso de forma conjunta.

\section{REFERENCIAS BIBLIOGRÁFICAS}

1. Álvarez. J. (1996). Contabilidad de Gestión Avanzada. McGraw-Hill Interamericana, Madrid, España.

2. Apaza. M. (2005). Costos ABC, ABM y ABB. Editora y Distribuidora Real SRL, Lima, Perú.
3. Bellido. P. (2003).Activity Based Costing-Costeo Basado en Actividades. Instituto de Investigación El Pacífico, Lima, Perú.

4. Horngren. C. (2007). Contabilidad de Costos. Ed., Prentice Hall Hispanoamericana S.A, Naucalpan de Juárez, México.

5. Hicks. D. (1998). El sistema de costos basado en las actividades (ABC). 1 era Ed., Alfaomega / Marcombo, México D.F, México.

6. Polimeni. R. (2000). Contabilidad de Costos. 3. a McGraw-Hill Interamericana S.A., Bogotá, Colombia.

7. Romero. A. (1993). La Contabilidad Gerencialy los nuevos métodos de costeo. Instituto Mexicano de Contadores, México D.F, México.

8. Santa Cruz.A. (2008). Tratado de Contabilidad de Costos. Tomo II. Instituto Pacífico, Lima, Perú.

9. Torres. G. (2006). Tratado Contabilidad de Costos por Sectores Económicos. Consultores S.A, Lima, Perú. 